\title{
The Learning Outcomes of an Australian Social Work Student's Study Tour to India
}

\author{
Pat Dorsett* and Julie Clark ${ }^{\dagger}$
}

\begin{abstract}
This article will explore the impact and learning outcomes of a short term study tour of a cohort of Australian social work students. Of particular interest was the impact of the programme on the students and specifically knowledge of international practice, cultural sensitivity as well as personal and professional growth. A qualitative approach was adopted to provide insights into the perceived impact of the programme and its contribution to the preparation of social work students to work in an increasingly globalised world. Indications are that it was effective in enhancing cultural sensitivity, understanding of structural factors contributing to inequity, the practical experience of poverty, personal growth and professional identity. For students, it was a valued and transformational learning experience. The "real life" experiential learning was highly valued by students.
\end{abstract}

Keywords: India; Study abroad, Internationalisation; Social work

* School of Human Services and Social Work, Griffith University, Queensland, Australia; p.dorsett@griffith.edu.au

$\dagger$ Former Lecturer, School of Human Services and Social Work, Griffith University, Queensland, Australia; j.clark@griffith.edu.au 


\section{Introduction}

Internationalisation has come to play an increasingly important role in social work curriculum both in Australia and overseas (Kreitzer, Barlow, Schwartz, Lacroix, \& Macdonald, 2009) Mathieson and Lager 2007, Nagy \& Falk 2000, Healy 2001, Panos et al. 2004). In the current climate of globalisation and international resettlement, social work graduates must develop competencies to work locally with issues arising from international mobility including work with migrants, asylum seekers, refugees as well as in overseas contexts (Payne \& Askeland, 2008). These imperatives have meant that social work educators are increasingly seeking to internationalise their curricula by incorporating international perspectives, awareness of culturally diverse populations, cultural sensitivity, and self-awareness of personal and traditional preconceptions (Dominelli \& Hackett, 2011; Mukherjee \& Chowdhury, 2012).

The most common strategies to incorporate international perspectives in social work curricula have included; embedding international content into existing courses, creating specific courses, and overseas study tours or international field practica (Mukherjee \& Chowdhury, 2012; Nuttman-Shwartz \& Berger, 2012). It is reported that one in five accredited social work schools in the USA include options for international practica in their programmes (Panos, Pettys, Cox, \& Jones-Hart, 2004). Research into the impact or outcomes of these activities has however, not kept pace with these initiatives (Kreitzer et al., 2009; Lough, 2009). In the Australian context, reports of internationalisation strategies within social work curriculum are limited as are evaluations of these strategies (Bell, Moorhead, \& Boetto, 2015; Fairchild, Pillai, \& Noble, 2006). Pawar, after an extensive review of the literature, concluded that "there is very little discussion of international social work practice and students perspectives in the Australian literature" (Pawar, Hanna, \& Sheridan, 2004, p. 224).

This paper explores outcomes of a two-week study tour by a group of Australian social work students undertaking a facilitated programme of study in Bangalore, India. Student evaluations and reflections provided rich source of qualitative data to explore the 
learning experiences and impact of the programme. Indications are that the study tour was effective in enhancing cultural sensitivity, cultural knowledge, understandings of the structural factors and professional identity. For some students it was a transformational experience. The "real life" experiential learning was highly valued by students. However, before exploring the student outcomes, an overview of the relevant literature, a description of the programme and the evaluation strategy are presented.

\section{Literature Review}

Reported outcomes from international social work student experiences include: personal growth; consolidated professional identity (Kreitzer et al., 2009; Nuttman-Shwartz \& Berger, 2012); cultural competence/sensitivity (Gilin \& Young, 2009; Krause \& Broderick, 2005; Kreitzer et al., 2009; Nuttman-Shwartz \& Berger, 2012); and increased understanding of the impact of poverty, oppression and human rights (Bell et al., 2015; Gilin \& Young, 2009; Kreitzer et al., 2009; Larson \& Allen, 2006; Saunders, Krause, \& Focht, 2012; Wehbi, 2009).

The potential for international study experiences to facilitate personal growth and consolidated professional identity is identified as an important outcome (Gilin \& Young, 2009; Kreitzer et al., 2009; Nuttman-Shwartz \& Berger, 2012). A Canadian study reports that all students participating in a social work cross-cultural exchange reported impact on both-their commitment to the profession and their personal values and beliefs (Kreitzer et al., 2009). They also reported an enhanced confidence in their ability as a social worker and respect for the social work profession.

A number of studies report a positive and significant relationship between studying abroad and cultural competence (Krause \& Broderick, 2005; Kreitzer et al., 2009; Nuttman-Shwartz \& Berger, 2012). Research indicates three key areas of cultural competence which are enhanced through overseas study experiences. These include: becoming more aware of one's own culture (and biases); gaining knowledge of cultural difference and developing skills to be more culturally sensitive and culturally appropriate when dealing with people from different backgrounds (Saunders et al., 
2012). Gilin and Young (2009) and Kreitzer et al., (2009) further highlight the experience of being "the other" in a different culture as an insightful learning experience contributing to resilience and increased empathy. All articles reviewed indicated that students were more confident with regard to their capacity to work in multicultural environments. The reports of enhanced cultural competence are largely based on students' qualitative reflections on learning. The need for future research adopting objective measures of change is recommended in the literature (Kemp \& Krause, 1999).

When students observe and experience the connections between poverty, inequity, discrimination and the socio-political practices in an environment different from their own, it can contribute to clarification of their own values and understandings in a way that could not be replicated in a classroom (Gilin \& Young, 2009). Larson and Allen (2006, p. 511) describe "an intense transformation... noting a significant shift in [students'] thinking from attributing individual responsibility for poverty and disadvantage towards broader structural factors". It has been suggested that a genuine understanding of the role of power in majority-minority relations is a key to developing greater sensitivity and cross cultural empathy (Saunders et al., 2012). To challenge superficial cultural stereotypes it is important that students are able to critically engage with the interplay between poverty, disadvantage and contextual factors inherent in the sociopolitical structures (Wehbi, 2009).

Critical reflection is integral in international social work learning experiences to enhance cultural sensitivity, empathy, and personal growth (Gilin \& Young, 2009; Larson \& Allen, 2006; NuttmanShwartz \& Berger, 2012). Journaling and guided debriefing exercises are the most frequently reported tools to facilitate critical reflection (Larson \& Allen, 2006; Nuttman-Shwartz \& Berger, 2012). Critical reflective journals are often integrated into formal student assessment of international courses (Larson \& Allen, 2006). Das and Anand (2000) provide a detailed description of strategies for critical reflection in international contexts for social work students utilising a paired peer approach. Critical reflection "has been found to potentially uncover many unconscious assumptions that directly influence and inform our work. Gaining this insight allows 
practitioners to consciously change their practice to be more in line with their desired ideological framework/intentions" (Morley, 2004).

The importance of institutional support as well as the challenges in implementing international programmes is highlighted in the literature (Gilin \& Young, 2009; Kreitzer et al., 2009; Lough, 2009; Nuttman-Shwartz \& Berger, 2012; Panos et al., 2004; Pawar et al., 2004; Saunders et al., 2012). A high level of planning and administrative support often places strain on the resources of schools of social work and faculty. Some social work programmes have established and maintain ongoing relationships with international partners to facilitate and support international study options (Rai, 2004) and a small number of programmes have reciprocal arrangements which supports the movement of students and staff in both directions (Kreitzer et al., 2009; Saunders et al., 2012). Panos et al., (2004) reported that the most active international social work programmes demonstrated a high level of commitment to developing and maintaining relationships in the overseas host institutions. To do this effectively places additional demands on institutional resources (Kreitzer et al., 2009; Nuttman-Shwartz \& Berger, 2012).

Adequate preparation of students prior to departure for an overseas destination is considered crucial (Kreitzer et al., 2009; Nuttman-Shwartz \& Berger, 2012; Pawar et al., 2004). Likewise, the need for onsite support, debriefing and supervision is highlighted (Kreitzer et al., 2009; Larson \& Allen, 2006; Nuttman-Shwartz \& Berger, 2012; Rai, 2004). Both formal and informal support was considered central to the outcomes for students (Pawar et al., 2004). This has implications for the academic staff accompanying students, in terms of the personal demands on their time and energy (Krause, 2000), particularly when programs are intense and fast paced.

\section{Overview of the India Gateway Program}

The India Gateway Program (IGP) was introduced in 2010 at Griffith University as an educational activity offered by the School of Human Services and Social Work in partnership with Christ 
University, Bangalore, India. The partnership and opportunities have evolved over the last five years, as have the relationships between academic staff from both Universities. Opportunities for student practicum experiences, involvement in conferences for students and staff, volunteering with projects, sharing and development of teaching philosophies, practices and resources have resulted from the maturing relationships. To date, this has largely involved Australian students and academic staff travelling to India. Opportunities for two way staff and/or student exchanges are currently being explored.

In 2012, Griffith University introduced a course, "Working in International Communities" as part of their social work programme. The aim of the course was to introduce students to social, political and cultural contexts of another country and to enhance understanding of national and international responses to contemporary social issues. Participation in the IGP, two-week study tour to India, was a key requirement of the course. The IGP provided opportunities to enhance cultural sensitivity and allowed students experience first-hand, the reality of social situations outside of Australia. This makes real the notion of social work in a globalised world and enables students to gain an understanding of the challenges that are faced by different countries. The opportunity to travel and be immersed in another culture allows students to make comparisons and develop their understandings of international obligations, goals and challenges in a globalised community.

In India, students visited non-governmental organisations providing services for vulnerable groups such as people with disabilities, "rescued" street children, and marginalised communities, as well as a number of hospitals. They visited small businesses and multinational corporations actively engaged in corporate social responsibility activities. They travelled to a village and participated in community development activities (such as social mapping) alongside community members. In addition, there were opportunities to visit sites of cultural significance such as places of worship, places of historical and social significance and to experience traditional rituals and dance. Both formal and informal 
interactions between IGP participants and Indian University staff and students enabled individuals to build relationships.

Throughout the IGP, students were encouraged to adopt a critical reflective stance to understand their experiences and were introduced to a framework for critical incident analysis in a workshop prior to departure (Fook, White, \& Gardner, 2006; Payne \& Askeland, 2008). Student diaries were structured to encourage the recording of critical incidents as they were encountered in the field and to capture some initial reflection "in situ". These incidents were the substance of both personal reflection and group debriefing sessions, which provided the opportunity to explore both at an intellectual level and at an affective level, the experience or situation. Critical reflection also was a part of the day-to-day informal interactions between students and with academic staff. These informal discussions often occurred when walking from place to place, travelling in the bus or over shared meals. The informal interactions were powerful means to reflect on and challenge personal values, underlying assumptions and the influence of culture, poverty and power. Formal group debriefing sessions allowed for the sharing of these insights and opportunities, for staff and students to critically deconstruct the situations experienced together. The critical incident analysis framework has been found to be useful in developing and contextualising professional knowledge, competence and enhanced self-awareness by challenging preconceived values and assumptions (Das \& Anand, 2012; Payne \& Askeland, 2008). After returning to Australia, students wrote a more formal critical reflective essay of their learning, drawing on the observations recorded in the travel diary.

\section{Evaluation Methodology}

As this was a new programme, an evaluation of the IGP was undertaken. The approach described by Wadsworth (2011) as an "everyday evaluation on the run", that is, an evaluation which is built into the work already being undertaken. Of particular interest was the impact of the programme on the students and specifically knowledge of international practice, cultural sensitivity as well as personal and professional growth. For this reason, a qualitative 
evaluation methodology was utilised and the voice of the students was privileged in describing the impact of the IGP experience for them. We have, therefore, given priority to the voice of the student while reporting the findings and the meanings they have attached to the experience growth (Cherin \& Meezan, 1998; Hoggarth \& Comfort, 2010; Wadsworth, 2011).

\section{Participants}

The IGP was advertised to students via the School website and during teaching sessions on campus. Twenty-two students applied and were accepted into the IGP in 2012. Students were aged from 19 to 54 years. This meant that the students had a range of life and educational experiences prior to commencing IGP.

Two academic staff travelled with the students to support their learning and facilitate individual and group debriefing as described above.

\section{Ethical considerations}

The evaluation research methodology was reviewed and approved by the Griffith University Human Research Ethics Committee in accordance with the Australian "National Statement on Ethical Conduct in Human Research" (2007). Participation in the evaluation was voluntary and not linked to any coursework assessment. All data provided by the students was de-identified and reported in a way that ensured confidentiality.

\section{Data collection}

A questionnaire consisting of seven open-ended questions to explore the student perception of learning and the personal impact of the IGP experience was developed by the authors for this evaluation. The questionnaire was administered in the first week of the programme and again one month after the completion of the IGP. Questions such as those below were incorporated into the questionnaire to encourage students to reflect on their learning outcomes and to provide examples from their experiences.

"During the IGP what (if anything) challenged you or changed for you? (e.g. attitudes, values, knowledge, awareness of culture etc.) Give an example if possible." 
"As you have reflected on the IGP experience since returning from India, have you continued to be challenged or changed by the things you experienced during the IGP? If so, how?"

Seventy-three per cent $(n=16)$ of the students returned the week 1 questionnaire, and $41 \%(n=10)$ returned the final questionnaire. The response rates were perhaps influenced by a number of factors. Completion of the survey was voluntary and the busyness of returning home to the commencement of a new semester may have contributed to the lower response rate for the post IGP survey.

Two staff accompanied the group to provide co-ordination, group de-briefing and support for learning. Group debriefing and informal interactions helped to create a shared understanding of how individuals were making sense of the experience and allowed some challenging of the individual constructions of meaning. Issues of culture, one's own and understanding of "others"' cultures often formed the basis for discussion. As the group composition included people from a range of cultures, the discussion was personal and challenging to negotiate for some group members. It seemed, perhaps for the first time; immersion in India had enabled some group members to appreciate how "being Australian" was perceived differently by each member of the group and thus the experiences in India were also qualitatively unique. For example, Australian students, of Indian heritage, expressed feeling both the same as and different from the majority of people around them.

\section{Data analysis}

The feedback data was de-identified and collated into a single document with responses to each question grouped together. The data was then analysed using thematic analysis techniques (Coffey \& Atkinson, 1996). The first author read each student's responses and developed codes. These codes were then clustered into themes which scanned across all of the responses to offer an account of overall student experience. To ensure consistency and integrity of the findings, the second author independently reviewed the coding of the data. Any discrepancy in consensus was resolved through 
discussion. Both researchers identified the recurrent themes across the initial concepts to produce a set of five themes that were derived from the data. Substantial portions of the student data is reported in the findings to support the interpretations of the findings (Dey, 1993; Locke, 1998; Taylor \& Bogdan, 1998).

\section{Findings}

Five themes were highlighted in the data including: cultural sensitivity; poverty; the role of governance and structural issues; self-awareness; and enhanced social work professional identity.

\section{Cultural sensitivity}

Enhanced cultural sensitivity was the most prevalent expectation of students when they commenced the IGP. Ninety four per cent (94\%) of students who responded to the first round of surveys $(n=15)$ indicated an expectation that they would gain enhanced; 'cultural awareness', 'knowledge about working with cultural differences' and 'cultural sensitivity' by participating in the programme.

\section{"I hoped to be exposed to major cultural differences which I would need to grapple with. I wanted to be immersed in the culture." \\ "...to see hear and feel the culture, current situation first hand, and gain a knowledge of India."}

The first week of the programme proved somewhat thoughtprovoking for some students who indicated that they were experiencing challenges to their prior understanding as well as their capacity to respond to their experiences in India. Some students felt discomforted and this seemed to provide the environment for shifts in thinking:

"...it was a challenge to strive to be culturally sensitive in some instances and I definitely have a changed attitude in terms of values and beliefs of various cultures."

As the study tour continued, students, consistent with their expectations, reported the most significant learning related to culture and the differences between the Indian and Australian 
social arrangements. The immersion experience proved to be powerful in helping students to move beyond the formal teaching about cultural sensitivity received in the classroom prior to departure:

"I have learnt to be more sensitive to other cultures and religions which I think is a massive learning for me as ...in this type of work we will come across many different cultures and it is great to be mindful of that."

At the conclusion of the IGP, a number of students perceived they had gained increased depth to their cultural awareness which would contribute to changes in their social work practice, with some students reporting significant attitudinal changes.

\begin{abstract}
"Furthermore, I believe my cultural competence has increased immensely. I have travelled to many different countries before but I have never been to a country with such stark contrasts to Australia.... This trip has taught me to be more open-minded, relaxed and patient as this seems to be the Indian way of life."
\end{abstract}

By the end of the programme, students were reporting that consistent with their expectations, the most significant learning was related to culture. The lived experience was proving to be powerful in helping them to understand the realities of cultural sensitivity in a way that could be associated with practice integration.

\title{
2. Poverty
}

For many students the extent of poverty and its influence on the health and well-being of people was particularly impactful. They observed and experienced a range of emotional and cognitive shifts which they sought to integrate into their knowledge and practice base for future reference.
"... seeing the extent of the poverty... People living in the equivalent of a one-man tent ... with little protection from the elements. People sleeping on the ground in the open air. Maybe my world is too sterile but the environment here makes me really sad for the people and I feel guilty."

"I was challenged by the large differences between the rich and poor and the disparities throughout the entire city. Also it was a 
challenge to strive to be culturally sensitive in some instances and I definitely have a changed attitude in terms of values and beliefs."

For some students the extent of poverty and the obvious contrast of the wealth that was so evident in India, created a challenging experience and some inner tensions as they grappled to make sense out of their observations. The observations led them to consider issues of social justice and human rights.

\section{Role of governance and structural issues}

One of the goals of the IGP was to create opportunities for students to explore and compare socio-political arrangements that were different from Australia. Students were encouraged to reflect on their experiences to develop their own understandings of the inherent tensions in international obligations, goals and challenges in a globalised community. Students noted the disparity between the role of government in Australia and the role of government in India and the importance of government in addressing social inequities and human rights.

I have learnt of my great fortune in being born into such a different society which has strong government regulation and small population in comparison to India. I feel that every person's small contribution can make a difference.

I have also learnt that government $\mathcal{E}$ policy have a huge impact on what can and can't be achieved both socially and from a health and well-being perspective.

Students became increasingly aware of the complexity of social situations and that their own preconceived and at times, idealistic perceptions about solutions may not always be the most appropriate.

"[I realised that] ...it is not as easy as saying 'let's change the world', 'let's help the children' - there are a lot of other differences that need to be thought about."

Some students experience confronting emotional reactions to their observations. Staff accompanying the group was able to offer support to process these reflections and help students achieve a better understanding of the structural factors that may contribute 
and the complexity and tensions inherent in developing effective and sustainable interventions.

\section{Self-awareness}

The context of the IGP provided an environment that facilitated personal growth. All student participants reported greater awareness of their own pre-conceptions, changed values/attitudes and personal growth. Specifically students reported a greater valuing of knowledge other than their own and increased selfawareness of their own underlying values, assumptions and potentially judgemental attitudes as demonstrated in the data presented below:

"The trip has given me the ability to view social issues in a new way. I now understand that before drawing a conclusion on any topic I need to gain more knowledge. Each situation is different. I have to be more mindful and sensitive to others' needs."

"I have learnt that I have had a lot of misconceptions about India and ... It has really surprised me so I have learnt humility. It changed my views about what is important and taught me that you don't need much to do a lot. There are people with nothing doing so much to help others."

There were a number of students who had not travelled overseas before and who had not been separated from their families before. For them the IGP proved to be a powerful personal growth journey. Some found the confrontations of the IGP experience particularly challenging. However, as they "pushed through" these challenges they were able to achieve significant personal growth.

At times, I was confronted with challenging situations, but I realised I have become more patient and tolerant. My knowledge base has increased immensely on the plight of the poor. Also being aware of my own strengths and weaknesses when out of my comfort zone.

For some students the shift in values and personal growth was transformative as reflected below: 
"I don't think my life will ever be the same again. I will think twice before making judgements/ assumptions and be far more willing to listen empathetically. I will also understand better that each person's experience is individual to them and as a practitioner one of my main roles will be as a facilitator of change ... All people have worth and deserve to be treated with respect."

\section{Enhanced Social Work professional identity}

The IGP, like many other experiential learning opportunities, provided a rich environment for students to clarify their professional goals, gain confidence and a greater sense of competence. Several students reported that this experience had confirmed that they had chosen the right profession and/or helped them find direction as emerging professionals.

\section{“...there are issues surrounding their [rural villagers'] poverty but these people have made me look at the plenty I have and enjoy as the liberties and freedom and it makes me want even more to work in the social work profession."}

In addition, the students reported a heightened awareness of social justice, human rights issues as well a commitment to social change processes to address these concerns.

"My attitude has definitely changed regarding the possibility of making positive difference. It was inspiring to see the huge difference and contributions that Christ University students made to the community and the progress and achievement of their work. I most definitely have increased interest in community development."

For $30 \%$ of the student participants, it underscored their interest in working in an international setting.

"I learnt that I have the ability to work in international communities - something that I thought would be too far out of my comfort zone before I left."

"I think it [IGP] has changed my future career completely. The programme has really made me think 
what I want to do with my future and how can I incorporate my career with doing something meaningful to make a difference in developing countries. I am dedicating one day of my week to volunteer/ community work ...I hope that this will give me the basic skills I require to take this further."

For others, it was about a greater awareness of themselves as developing professionals.

"As a developing professional, [I] feel I've had the
opportunity to develop my skills to work alongside
different communities etc., and my understanding of
community empowerment has been heightened. As a
social worker I believe I will come across people of all
religions/ cultures with various values and beliefs and
this trip has given me the tools to be able to do this in an
appropriate professional manner.

It [IGP] will influence the way I perceive things. To see the people in the bigger picture, I will take more holistic approach to practice. I strongly believe in autonomy and self-determination. Utilise the skills clients possess and empower them to achieve their goals."

\section{Discussion}

This evaluation highlights the potential for international short term study tour experiences to deepen student learning about the international context of social work, develop cultural sensitivity, the impact of poverty and structural issues and governance. Students developed personal insights contributing to both individual and professional growth as well as an enhanced sense of competence for practice. The students consistently described an intense personal experience contributing to a transformation in their understanding of the impact of poverty and disadvantage on health and welfare of people, the structural implications, and insights into their own personal values and prejudices. A key theme emerging from the data suggests significant personal growth, enhanced professional confidence and cultural sensitivity. 
These findings are consistent with findings reported in similar programmes and in the literature (Larson \& Allen, 2006).

The IGP study tour was fast-paced and our experience suggests that adequate time needs to be built into the programme for reflection and debriefing. In a structured environment with a facilitated inclusive discussion as well as some considered confrontation and knowledge-sharing, useful and transformative learning can occur. As the learning may not be in "real" time for all students, it is important for staff travelling with the group to be informally available to students, who are still processing their experiences, after the conclusion of the travelling component of the programme. The whole group debriefing sessions are important in assisting students to consolidate their day-to-day experiences during the programme. It is important to take into account the individual needs of students.

It is acknowledged that this is a single study based on a small sample. While the results cannot be generalised, they do provide initial evidence of positive outcomes. It is recommended that future evaluations should incorporate a pre and post design to quantitatively measure changes in cultural awareness as a key outcome of such programmes. Undertaking similar research with students from the host university could provide understanding of the reciprocal influence of such programmes.

Study tours expose students to social arrangements that can be considered in contrast to their own experiences. They offer opportunities to engage, reflect and ask questions in an environment with a different level of intensity to other teaching environments. Within the context of an international partnership, as has been established with Christ University, the opportunity can make significant contributions to students' professional development in a range of areas as we have discussed. A study tour such as the India Gateway Programme, described in this article, is one way to promote an international perspective in learning for social work students. While recognising the limitations of this study the authors remain convinced that international social work experiences are powerful educational strategies to facilitate transformative learning and critical reflection. International programmes such as the IGP offer opportunities to enhance 
students' preparation to work with cultural diversity and to better understand the basis for similarities and differences in social arrangements.

\section{Acknowledgments}

The IGP is not possible without the generosity and good will of many staff members of Christ University, in particular, the Office of International Affairs and the organisations that partner with them to offer such a rich experience.

\section{Funding Acknowledgements}

This tour was supported by a grant from Griffith International and the Australian Commonwealth Government to subsidise travel costs for students.

\section{Declaration of Conflicting Interests}

There are no conflicts of interest to declare.

\section{References}

Bell, K., Moorhead, B., \& Boetto, H. (2015). Social work students' reflections on gender, social justice and human rights during a short-term study programme to India. . International Social Work. doi: 10.1177/0020872814559559

Cherin, D., \& Meezan, W. (1998). Evaluation as a Means of Organizational Learning. Administration in Social Work, 22(2), 121. doi: DOI: $10.1300 / J 147 v 22 n 02 \_01$

Coffey, A., \& Atkinson, P. (1996). Making Sense of Qualitative Data. California: Sage.

Das, C., \& Anand, J. C. (2012). Strategies for critical reflection in international contexts for social work students. International Social Work. doi: 10.1177/0020872812443693

Dey, I. (1993). Qualitative data analysis: A user-friendly guide for social scientists. London.

Dominelli, L., \& Hackett, S. (2011). Internationalizing social wotk theory and practice (Editorial). International Social Work, 55(2), 151-153. 
Fairchild, S. R., Pillai, V. K., \& Noble, C. (2006). The impact of a social work study abroad program in Australia on multicultural learning. International Social Work, 49(3), 390-401. doi: 10.1177/0020806063413

Fook, J., White, S., \& Gardner, F. (2006). Critical Reflection: A Review of Contemporary Literature and Understandings. In S. White, Fook, J. and Gardner, F. (Ed.), Critical reflection in health and social care (pp. 3-20): McGraw-Hill International.

Gilin, B., \& Young, T. (2009). Educational benefits of international experiential learning in an MSW program. International Social Work, 52(1), 36-47. doi: 10.1177/0020872808093347

Hoggarth, L., \& Comfort, H. (2010). A Practical Guide to Outcome Evaluation. London: Jessica Kingsley Publishers.

Kemp, B. J., \& Krause, J. S. (1999). Depression and life satisfaction among people ageing with post-polio and spinal cord injury. Disability And Rehabilitation, 21(5-6), 241-249.

Krause, J. S. (2000). Aging after spinal cord injury: an exploratory study. Spinal Cord, 38(2), 77-83.

Krause, J. S., \& Broderick, L. (2005). A 25-year longitudinal study of the natural course of aging after spinal cord injury. Spinal Cord, 43(6), 349-356.

Kreitzer, L., Barlow, C., Schwartz, K., Lacroix, M., \& Macdonald, L. (2009). Candian and EU social work students in a cross-cultural program: What they learnt from the experience. International Social Work, 55(2), 245-267.

Larson, G., \& Allen, H. (2006). Conscientization - the experience of Canadian social work students in Mexico. International Social Work, 49(4), 507-518. doi: 10.1177/002087280605327

Locke, S. (1998). Qualitative research and data analysis. British Journal of Therapy and Rehabilitation, 5(7), 357 - 361.

Lough, B. J. (2009). Principles of effective practice in intenational social work. Journal of Social Work Education, 45(3), 467-480.

Morley, C. (2004). Critical reflection in social work: A response to globalisation? International Journal of Social Welfare, 13, 297-303.

Mukherjee, D., \& Chowdhury, D. (2012). What do the flyers say? Embedded 'Orientalist' constructions in social work study abroard programs in the Unitied States. International Social Work, 1-21. doi: 10.1177/0020872812441644 
Nuttman-Shwartz, O., \& Berger, R. (2012). Field education in international social work: Where are we and where should we go. International Social Work, 55(2), 225-243. doi: 10.1177/0020872811414597

Panos, P. T., Pettys, G. L., Cox, S. E., \& Jones-Hart, E. (2004). Survey of international field education placements of accredited social work education programs Journal of Social Work Education, 40(3), 467-478.

Pawar, M., Hanna, G., \& Sheridan, R. (2004). International social work practicum in India. Australian Social Work, 57(3), 223-236. doi: http:/ / dx.doi.org/10.1111/j.1447-0748.2004.00150.x

Payne, M., \& Askeland, G. (2008). Globalization and International Social Work. Ashgate: Aldershot.

Rai, G. S. (2004). International field work experience: A survey of US schools. International Social Work, 47(2), 213-226.

Saunders, L. L., Krause, J. S., \& Focht, K. L. (2012). A longitudinal study of depression in survivors of spinal cord injury. Spinal Cord, 50(1), 72-77. doi: 10.1038/sc.2011.83

Taylor, S. J., \& Bogdan, R. (1998). Introduction to Qualitative Research Methods: A Guidebook and Resource (3 ed.). New York: John Wiley \&Sons.

Wadsworth, Y. (2011). Everyday Evaluation on the Run: The userfriendly introductory guide to effective evaluation (3rd ed.). Crows Nest: Allen \& Unwin.

Wehbi, S. (2009). Deconstructing motivations: Challenging international social work placements. International Social Work, 52(1), 48-59. doi: 10.1177/0020872808097750 
Artha J Soc Sci

ISSN 0975-329X 